\title{
Conductivity Changes of Polyacetylene Exposed to $\mathrm{SO}_{2}$
}

\author{
Gerlinde Bischoff, Johann-Georg RaBE, Werner F. Schmidt, \\ and Katsumi YosHino* \\ Hahn-Meitner-Institut Berlin, Abteilung Strahlenchemie, \\ 1000 Berlin 39, F.R. Germany \\ * Department of Electronic Engineering, Faculty of Engineering, \\ Osaka University, Osaka, Japan
}

(Received November 24, 1992)

\begin{abstract}
The conductivity change of polyacetylene foils under exposure with different pressures of sulfur dioxide vapor was measured as a function of time. Adsorption and diffusion are the rate determining steps for the increase of the conductivity. A simple mathematical description of the two processes yields agreement with experiment.

KEY WORDS Polyacetylene / Sulfur Dioxide / Electrical Conductivity / Diffusion / Doping / Kinetics / Two Probe Method /
\end{abstract}

Samples of polymers with conjugated double bonds can be prepared as insulators. Their intrinsic conductivity is low and such samples are especially suited for the study of low level doping effects. Changes of the electrical conductivity induced by exposure to oxidizing or reducing gases may form the basis for the application of these polymers as sensors of toxic gases, such as $\mathrm{SO}_{x}$ or $\mathrm{NO}_{x}$. Previously, we have studied the conductivity changes induced by exposure of polypyrrole, polythiophene, and polyacetylene to various oxidizing or reducing gaseous compounds. ${ }^{1-3}$ In these studies, the conductivity change was measured as a function of vapor pressure. The results were discussed on the basis of a model which implied the adsorption of the vapor molecules coupled with an increase of the conductivity of the surface. ${ }^{3}$ Recently, time resolved measurements of the conductivity increase after exposure to various gases or vapors were carried out and a more detailed insight into the processes involved emerged. Here, we wish to report on the conductivity changes of polyacetylene exposed to sulfur dioxide.

\section{EXPERIMENTAL}

Polyacetylene was prepared according to the method developed by Ito et al. ${ }^{4} \mathrm{~A}$ strip of approximately $A=d \times h=5 \times 6 \mathrm{~mm}^{2}$ area and $\delta=60 \mu \mathrm{m}$ thickness was attached with silver paste to two tungsten wires serving as the electrodes. The electrode assembly was housed in a glass container which could be evacuated. The conductivity was obtained from measurements of the current, $I$, as a function of the applied voltage, $U$. A schematic diagram of the set-up is shown in Figure 1. Over a range of voltages a linear relationship between current and voltage was observed although sometimes the straight line did not go exactly through the origin. The slope $\Delta I / \Delta U$ was taken for the calculation of the conductivity $\sigma$, given as

$$
\sigma=\frac{\Delta I d}{\Delta U(\delta h)}
$$

The conductivities of the samples in vacuo were between $3 \times 10^{-8}$ and $2 \times 10^{-7} \Omega^{-1} \mathrm{~cm}^{-1}$. Sulfur dioxide was quickly admitted up to the desired pressure to the evacuated sample container and the current through the sample 


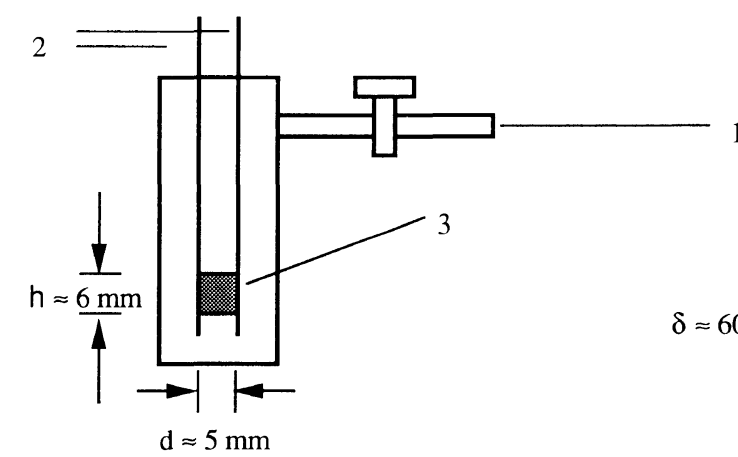

detail of the testcell: polymer film, rotated by $90^{\circ}$

1 -connection to vacuum

2 - tungsten electrodes

3 - polymer film, contacted with silverpaste



4 - DC-Power Supply (Delta Elektronika Mod. E0300-0.1)

5 - Electrometer (Keithley Mod. 617)

6 - Voltmeter (Prema Mod. 5000)

7 - Computer (Macintosh Mod. MacPlus)

8 - Testcell

Figure 1. Experimental set-up.

was measured as a function of voltage and time. Changing of the polarity of the voltage changed the polarity of the current but not its magnitude. This may serve as an indication that contact problems or inhomogeneity of doping were not encountered. In Figure 2, the current-voltage dependence of a polyacetylene sample after a $30 \mathrm{~min}$ exposure to $\mathrm{SO}_{2}$ is shown. Care has to be taken that the Joule heat dissipated in the sample does not lead to structural changes of the polymer. At $100 \mathrm{~V}$ a current of $2 \times 10^{-5} \mathrm{~A}$ was measured which gives a thermal load of the sample of approximately $1 \mathrm{~W} \mathrm{~cm}^{-3}$. The corresponding temperature rise should be of the order of $0.1^{\circ} \mathrm{Cs}^{-1}$. Convection cooling of the sample by the gas and heat flow to the tungsten wires will limit the temperature rise to a few degrees at most. Tests with a thermocouple did not show any temperature rise.

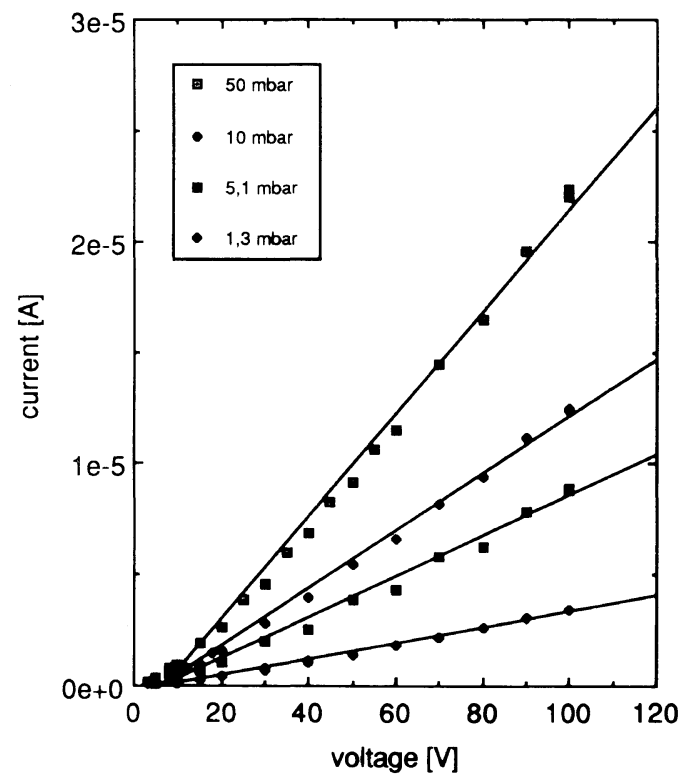

Figure 2. Current-voltage dependence of polyacetylene after $30 \mathrm{~min}$ exposure to $\mathrm{SO}_{2}$ gas. 


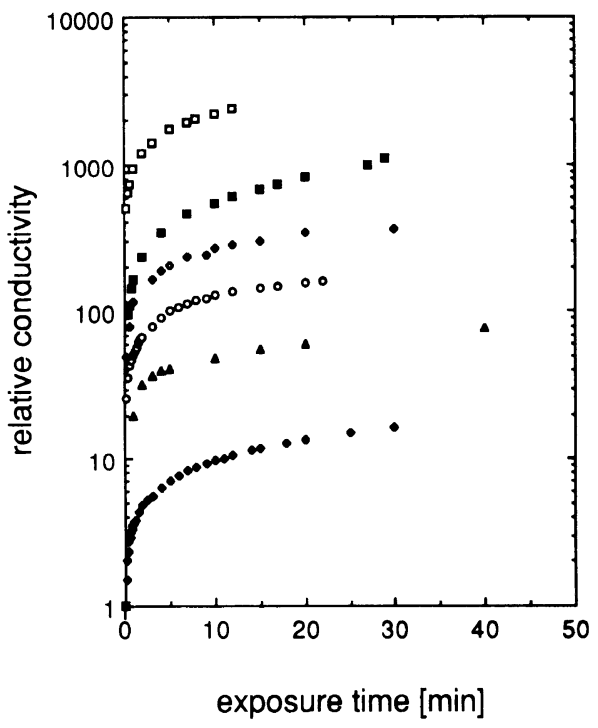

Figure 3. Evolution in time of the current through a sample of polyacetylene after exposure to $\mathrm{SO}_{2}: \diamond, 1.2$ mbar: $\triangle, 5.1$ mbar; $\bigcirc, 10$ mbar; $\diamond, 50$ mbar; $\mathbf{D}, 100$ mbar; $\square, 210$ mbar.

After exposure to a given pressure of $\mathrm{SO}_{2}$ the current increased fast within a minute. This region was followed by a more gradual increase. The data are shown in Figure 3. The increase in conductivity was irreversible. After evacuation of the sample for $120 \mathrm{~min}$ the conductivity remained constant and changed little if the sample was exposed to $\mathrm{SO}_{2}$ again. The maximum conductivity attained was of the order of $10^{-3} \Omega^{-1} \mathrm{~cm}^{-1}$ after $12 \mathrm{~min}$ exposure to 210 mbar. A stationary state of doping was not reached in these experiments. Thus no information on the concentration of dopant in the polymer can be given.

\section{DISCUSSION}

In conjugated polymers, the electric conductivity is enhanced by many orders of magnitude by doping with electron acceptor or donor molecules. However, the dynamic process has not been clarified as yet. Conductivity changes in organic semiconductors or insulators induced by gases or vapors

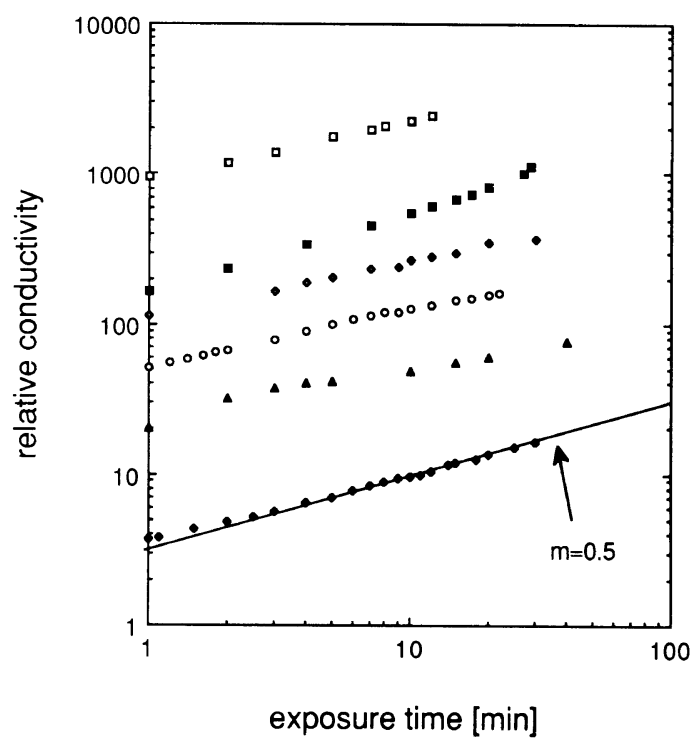

Figure 4. Double logarithmic plot of our data of Figure 3: $\diamond, 1.2$ mbar; $\triangle, 5.1$ mbar; $\bigcirc, 10$ mbar; $\diamond, 50$ mbar; $\square$ 100 mbar; $\square, 210$ mbar.

have been attributed to a modification of the surface conductivity. For insulators, weak chemisorption and formation of a charge transfer complex was invoked. ${ }^{5,6}$ If the gas molecules possess a dipole moment reduction of the band gap of the material near the surface and increased charge carrier formation is thought to occur. ${ }^{7}$ In addition to this surface effect, diffusion into the bulk of the material should take place connected with formation of additional charge carriers.

In the case of $\mathrm{SO}_{2} /$ polyacetylene, reaction of $\mathrm{SO}_{2}$ with the double bond of PA is thought to occur leading to the formation of $\mathrm{SO}_{2}^{-}$and $\mathrm{PA}^{+}$. Studies on electron attachment to three-atomic molecules containing oxygen have shown that dissociation of the initial negative ion with formation of $\mathrm{O}^{-}$is a likely process. ${ }^{8}$ Here, we would speculate that the reaction,

$$
\mathrm{SO}_{2}^{-} \rightarrow \mathrm{O}^{-}+\mathrm{SO}
$$

leads to the observed irreversibility of the conductivity change.

If we plot our data of Figure 3 in a $\log -\log$ plot (see Figure 4), the second time domain of 
conductivity increase seems to follow a $t^{1 / 2}$-dependence. Such a time dependence is characteristic of diffusion processes. Recently, Tansley and Maddison ${ }^{9}$ investigated the conductivity degradation in oxygen-aged polypyrrole. A decrease of the conductivity of doped polypyrrole is observed when the sample is exposed to atmospheric oxygen. Also in this case the change in time of the conductivity consisted of two regions which could be attributed to adsorption and diffusion.

The kinetics of the adsorption process can be described by the following differential equation,

$$
\frac{\mathrm{d} C}{\mathrm{~d} t}=\frac{C_{0}-C}{\tau}
$$

where $C$ denotes the actual concentration of molecules adsorbed at the surface and $C_{0}$ denotes the saturation concentration. $\tau$ is the characteristic time constant of the process. At time $t=0, C=0$ and the solution of eq 2 is obtained as,

$$
C(t)=C_{0}\left(1-\mathrm{e}^{-t / \tau}\right)
$$

We assume that the variation in time of the surface conductivity $\sigma_{\mathrm{s}}(t)$ is proportional to $C(t)$, i.e.,

$$
\sigma_{\mathrm{s}}(t)=K_{1} C_{0}\left(1-\mathrm{e}^{-t / \tau}\right)
$$

where $\sigma_{0}=K_{1} C_{0}$ denotes the maximum conductivity produced by a certain surface coverage at a given pressure. Generally, the dependence of the bulk conductivity of polymers on the dopant concentration is rather complex. From measurements on iodine/polythiophene, proportionality between bulk conductivity and dopant concentration may be inferred at low levels. ${ }^{10}$ Here, we are dealing with a conductivity, $\sigma_{\mathrm{s}}$, of surface layers induced by a small quantity of dopant. Proportionality of $\sigma_{\mathrm{s}}$ and $C$ is assumed for this case, too.

The diffusion of gas molecules into the material and doping of the polymer molecules in the bulk proceeds on a longer time scale. The diffusion process can be considered as one-dimensional with the coordinate $x$ directed perpendicularly away from the surface. The governing equation is given as,

$$
\frac{\partial C}{\partial t}=D_{\mathrm{b}} \frac{\partial^{2} C}{\partial x^{2}}
$$

$D_{\mathrm{b}}$ is the diffusion coefficient of $\mathrm{SO}_{2}$ in PA. The solution $C(x, t)$ of eq 5 is given by the complementary error function. The timedependent number density of infused species per unit surface area $N(t)$, is given as the integral, ${ }^{9}$

$$
N(t)=\int_{0}^{\infty} C(x, t) \mathrm{d} x=2 C_{0} \sqrt{D_{\mathrm{b}} \frac{t}{\pi}}=2 C_{0} \lambda
$$

In principle, the limits of the integral should be taken from 0 to the thickness of the sample $(60 \mu \mathrm{m})$. Since the diffusion coefficients of small molecules in crystalline organic solids are of the order of $10^{-10}$ to $10^{-12} \mathrm{~cm}^{2} \mathrm{~s}^{-1}$, the diffusion distance, $\lambda$, of the gas molecules into the film during the time interval of our experiment is of the order of $10^{-4}$ to $10^{-5} \mathrm{~cm}$ which is small compared to the thickness of the sample. This distance is not small when compared to the diameter of the fibrils $(20 \mathrm{~nm})$.

The infused molecules form charge transfer complexes with the polymer and thus produce an additional bulk conductivity $\sigma_{\mathrm{b}}$ which varies in time as,

$$
\sigma_{\mathrm{b}}(t)=K_{\mathrm{b}} C_{0} \sqrt{D_{\mathrm{b}} t}
$$

$K_{\mathrm{b}}$ is a constant. The total conductivity of the sample $\sigma_{\text {tot }}$, is given by the sum of the intrinsic conductivity $\sigma_{\text {vac }}$, the surface conductivity $\sigma_{\mathrm{s}}$ and the bulk conductivity $\sigma_{\mathrm{b}}$, i.e.,

$$
\sigma_{\mathrm{tot}}=\sigma_{\mathrm{vac}}+\sigma_{0}\left(1-\mathrm{e}^{-t / \tau}\right)+K_{\mathrm{b}} C_{0} \sqrt{D_{\mathrm{b}} t}
$$

In Figure 4 , the ratio $\sigma_{\text {tot }} / \sigma_{\text {vac }}$ is plotted as a function of time. The two time domains corresponding to adsorption and diffusion can be identified particularly well for the data of 210 mbar. In Figure 5 we compare the 




Figure 5. Comparison of the experimental data $(\bigcirc$ for 210 mbar $)$ and the dependence given by eq $8(\diamond, \tau=0.4$ $\left.\min ; \sigma_{0} / \sigma_{\mathrm{vac}}=411 ;\left(K_{\mathrm{b}} C_{0} \sqrt{D_{\mathrm{b}}}\right) / \sigma_{\mathrm{vac}}=568.5\left[\mathrm{~min}^{-0.5}\right]\right)$.

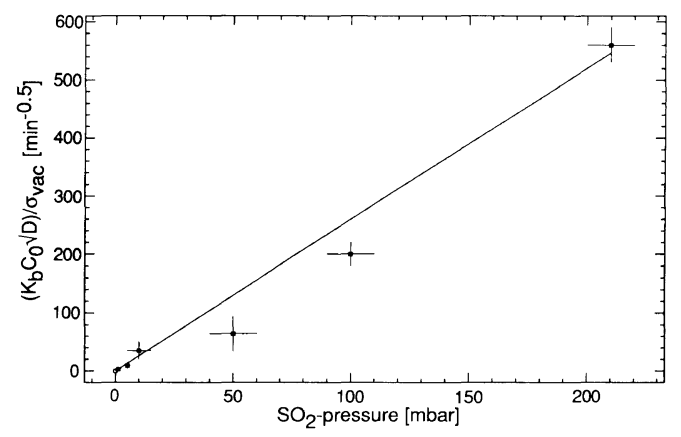

Figure 6. Slope $m^{*}$ (see eq 9) of the data of Figure 4 as a function of pressure.

experimental data with the dependence given by eq 8 . Adsorption approaches saturation with a time constant of $\tau \approx 0.4 \mathrm{~min}$ while for longer times diffusion becomes the rate determining step. For lower gas pressures, the separation in time of the two mechanisms is not so apparent. Since $\sigma_{\text {tot }} / \sigma_{\text {vac }}$ follows a $\sqrt{t}$-dependence over the whole time range measured we may assume that for lower pressures $\tau$ becomes smaller. At longer times, the increase of the conductivity is determined entirely by the diffusion term of eq 8 . The slope $m^{*}$ is given as,

$$
m^{*}=\frac{\left(K_{\mathrm{b}} C_{0} \sqrt{D_{\mathrm{b}}}\right)}{\sigma_{\mathrm{vac}}}
$$

From the data of Figure $4, m^{*}$-values were obtained. Since $D_{\mathrm{b}}$ and $K_{\mathrm{b}}$ are independent of pressure, $m^{*}$ is proportional to $C_{0}$, the surface coverage. In Figure $6, m^{*}$ is plotted as a function of pressure. In the pressure range investigated here, the surface coverage increases proportionally with pressure. Langmuir's adsorption isotherm predicts such a dependence for small pressures.

Whether the diffusion process observed here, corresponds to diffusion of the gas into the polymer fibrils or into the interior of the polymer foil which has a porous structure, formed by the fibrils, ${ }^{11-13}$ cannot be decided. Measurements on the recently developed $\mathrm{N}$-type film which has a high bulk density of 1.0 to $1.1 \mathrm{~g} \mathrm{~cm}^{-3}$ might answer this question.

Acknowledgment. The constructive remarks by the referees of this paper are gratefully acknowledged.

\section{REFERENCES}

1. K. Yoshino, H. S. Nalwa, J. G. Rabe, and W. F. Schmidt, Polym. Commun., 26, 103 (1985).

2. J. G. Rabe, W. F. Schmidt, and K. Yoshino, Jpn. J. Appl. Phys., 24, L583 (1985).

3. J. G. Rabe, G. Bischoff, and W. F. Schmidt, Jpn. J. Appl. Phys., 28, 518 (1989).

4. T. Ito, H. Shirakawa and S. Ikeda, J. Polym. Sci., Polym. Chem. Ed., 12, 11 (1974).

5. F. Gutmann and L. E. Lyons, "Organic Semiconductors," John Wiley \& Sons, New York, London, Sydney, 1967.

6. H. Meyer, "Organic Semiconductors," Verlag Chemie, Weinheim, 1974.

7. T. N. Misra, B. Rosenberg, and R. Switzer, J. Chem. Phys., 48, 2096 (1968).

8. L. G. Christophorous, "Atomic and Molecular Radiation Physics," Wiley Interscience, London, New York, Sydney, Toronto, 1971.

9. T. L. Tansley and D. S. Maddison, J. Appl. Phys., 69, 7711 (1991).

10. S. Hayashi, K. Kaneto, K. Yoshino, R. Matsushita, and T. Matsuyama, J. Phys. Soc. Jpn., 55, 1971 (1980).

11. H. Rommelmann, R. Fernquist, A. J. Epstein, M. Aldissi, T. Woerner, and P. Bernier, Polymer, 24, 1575 (1983). 
12. A. J. Heeger, in "Polyacetylene: New Concepts and New Phenomena," T. A. Skotheim, Ed., "Handbook of Conducting Polymers," Vol. 1, Marcel Dekker, New York and Basel, 1986, Chapter 21.

13. S. Pekker and A, Jánossy, in "Chemistry of Doping and Distribution of Dopants in Polyacetylene," $\mathrm{T}$. A. Skotheim, Ed., "Handbook of Conducting Polymers," Vol. 1, Marcel Dekker, New York and Basel, 1986, Chapter 2. 